\title{
Cells and Viruses at Molecular Resolution: From Structure to Mechanism
}

\author{
Sriram Subramaniam
}

National Cancer Institute, NIH, Bethesda, MD 20892

Emerging methods in 3D biological electron microscopy provide powerful tools and great promise to bridge a critical gap in imaging in the biomedical size spectrum. This comprises a size range of considerable interest that includes cellular protein machines, giant protein and nucleic acid assemblies, small subcellular organelles and bacteria. These objects are generally too large and/or too heterogeneous to be investigated by high resolution X-ray and NMR methods; yet the level of detail afforded by conventional light and electron microscopy is often not adequate to describe their structures at resolutions high enough to be useful in understanding the chemical basis of biological function. We are working towards obtaining an integrated molecular understanding of cellular architecture by combining novel technologies for 3D biological imaging with advanced methods for image segmentation and computational analysis. Recent efforts have been particularly focused on developing and applying these methods to image and model dynamic biological systems, with particular emphasis on applications to HIV/AIDS and cancer.

One area of research in the laboratory is directed towards understanding the mechanisms by which viruses are delivered to the surface of CD4+ T-cells, an essential component for developing effective strategies that can result in blocking HIV entry. Under physiological conditions, viruses arrive at the T-cell surface either by diffusion within the extracellular space, or by targeted delivery to specialized structures known as the virological synapse formed between the T-cell and antigen-presenting cells such as macrophages and dendritic cells. Using 3D electron microscopy, we have imaged the distribution of HIV at the virological synapse. Electron tomography of $200 \mathrm{~nm}$ thick sections and ion abrasion scanning electron microscopy of the entire cell-cell region (approximately 5 microns thick) reveal the presence of an intricate scaffold formed by cell surface protrusions from the dendritic cells and the CD4+ T-cells at the synapse. An extended network of virus-containing compartments is found inside the dendritic cell. Remarkably, these compartments appear to be connected to the virological synapse via channels that are about $100 \mathrm{~nm}$ wide that frequently contain viruses. We presume these viruses are in transit to the virological synapse. Similar channels are also found in virus-infected macrophages. Our results thus suggest a novel mechanism for delivery of HIV from the interior of antigen-presenting cells to CD4+ Tcells in "virion channels" that are likely to be much more effective at viral delivery then by the release of virus into the extracellular media via budding. Ongoing studies are focused on extending these structural studies to define the chemical composition and protein machinery that allows formation of the virological synapse, and the associated gradients of cellular components that are associated with its formation and maintenance. 


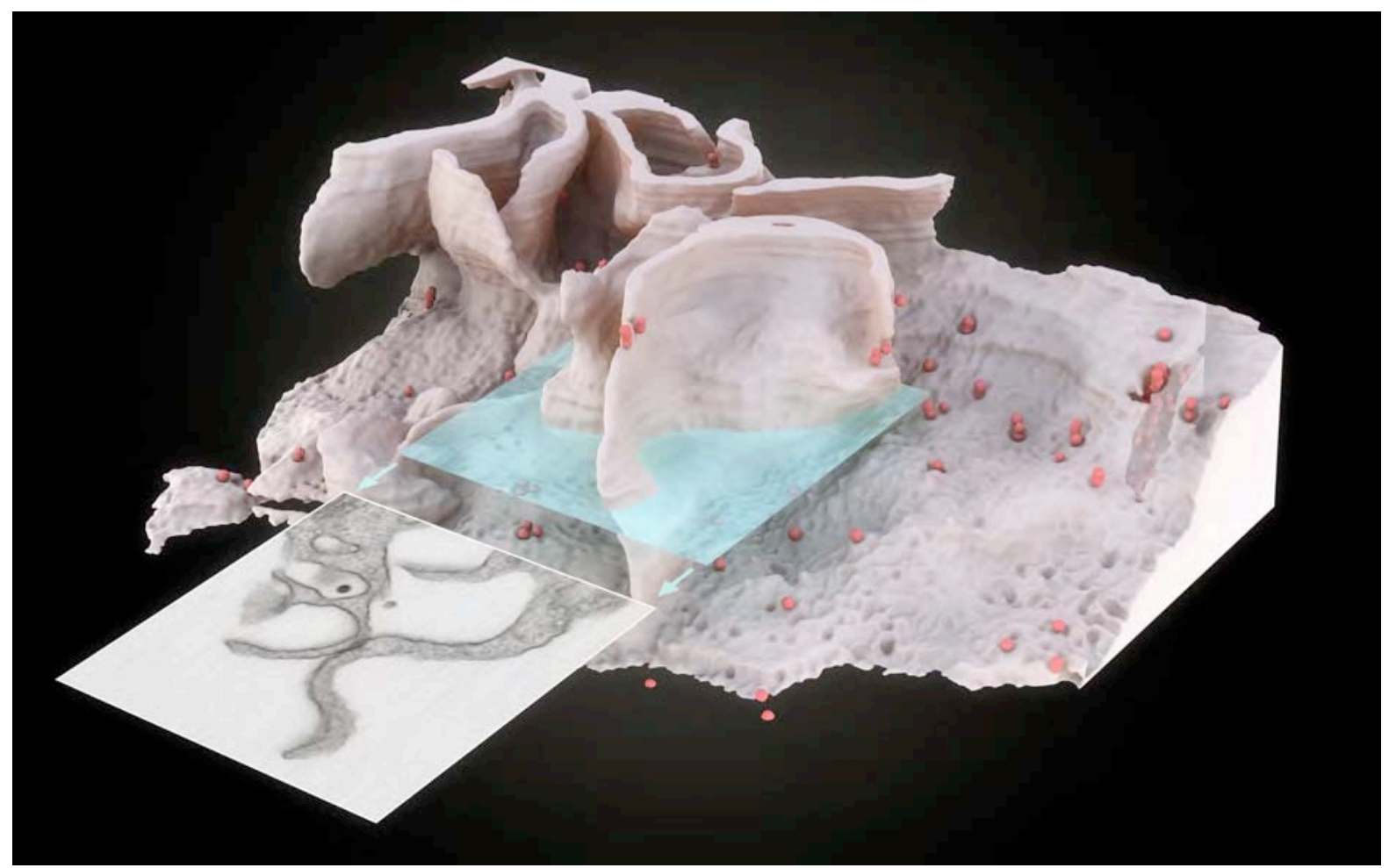

3D representation of the surface and interior of an HIV-infected macrophage obtai ned using newly developed tools for 3D imaging using ion-abrasion scanning electron microscopy (from Bennett et al (2009) PLoS Pathogens $\underline{\mathbf{5}}$, e1000591).
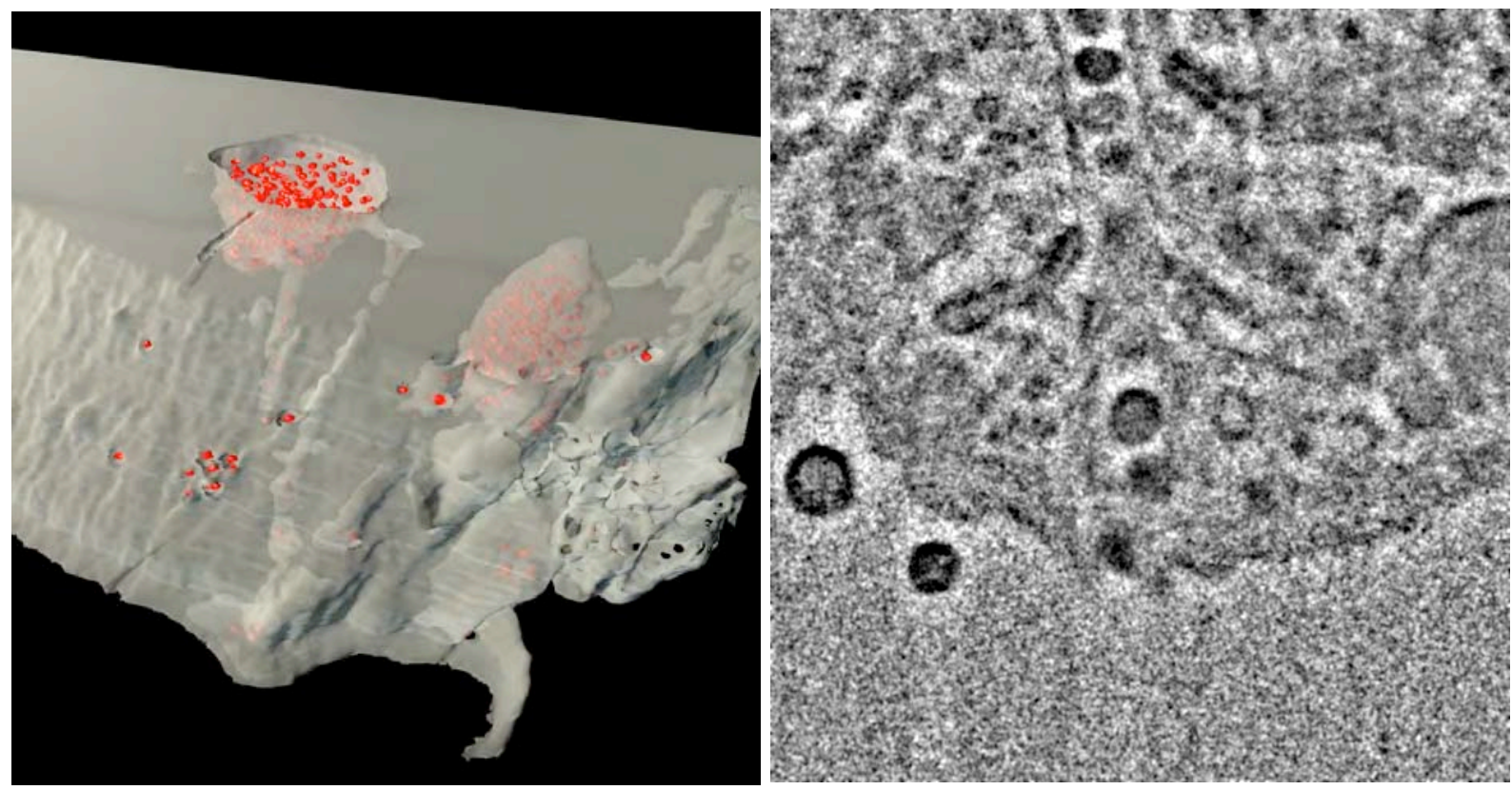

Left: 3D representation of the interior of a virus-infected macrophage. Narrow channels connect the intracellular compartments with the surface. The red spheres indicate individual HIV-1 particles in the cells that were imaged within these channels. Right: 2D image of a narrow HIV-1 lined channel near the surface of the macrophage (from Bennett et al (2009) PLoS Pathogens $\underline{5}$, e1000591). 